From our experience in the Presbyterian Hospital, this method is recommended as the safest and simplest method of conducting painless labor.

Its advantages are as follows :

1. The apparatus is simple, easily transported, and may be used by any practitioner.

2. Deep anesthesia is not necessary.

3. There are no ill-effects to mother or child.

4. The strength of uterine contractions is not diminished, no matter how long the administration of the nitrous oxid gas is continued.

5. The administration is under control all the time, and can be stopped at any moment. This is a very decided advantage which is not possessed by any method which necessitates placing a patient under the influence of drugs administered internally.

\section{NITROUS OXID GAS ANALGESIA IN OBSTETRICS}

\section{FRANK W. LYNCH, M.D. CHICAGO}

Nitrous oxid in anesthesia has become most popular during the last few years. Gas and ether machines have undergone marked improvements. Their use in surgery and dentistry is well-nigh universal. Analgesia has supplanted anesthesia in a considerable field of surgical work. Analgesia is obtained during the first stage of anesthesia. It is the stage of Dammerschlaf, the threshold of sleep, in which stimuli to the skin cause no mental impression, and consciousness is disordered.

Since July, 1913, I have used gas for long-continued analgesia in my obstetric work. The method should not be confounded with the old and well-known use of gas during the past generation, given to deeper stages of anesthesia about the time of actual birth. The results obtained by this new method have been astonishing. There is freedom from pain. There are no bad effects for mother or child. The gas in analgesic doses appears to stimulate the uterine pains. There is no doubt of the patient bearing down more energetically when under the influence of the drug. I made mention of the method at the Michigan State Medical Society at Flint, Sept. 1, 1913.1

I have used the method for more than one hour in thirty-four cases. Analgesia has been maintained from the latter part of the first stage, or from the time when the pains became severe; and all have said that pain was negligible and practically nil. There are twentyfive primiparas and nine multiparas in the series. Analgesia was continued in thirty-four cases more than one hour; in thirty-two cases more than two hours; in twelve cases more than three hours; in four cases more than four hours, and in one case more than six hours. Three cases were terminated with forceps with the gas carried to surgical degree. They were all primiparas, one of 39 years, one of 35 , and one of 25 , in whom there was transverse arrest of the head. There was no case of inertia, post-partum hemorrhage or shock.

The method is suitable for patients at their homes or in the hospital. Any gas machine with a Hewett stopcock permitting the admixture of oxygen and gas will do. I use a small portable machine, which holds four tanks. The best results are obtained with a nose-piece such as is used by dentists, but the ordinary mouth-piece will answer. I have hitherto started the treatment when the pains have become severe enough to occasion complaint. Pure nitrous oxid gas is turned full on at the beginning of the pain, and the patient is told to breathe deeply but rapidly through the nose. Five or six respirations suffice to produce analgesia, even in the presence of the uterine contraction. The nose-piece is now placed over the mouth. The patient is told to breathe through the mouth, and analgesia is maintained by admixing oxygen with the gas until the end of the pain. This process is repeated with each pain. The percentage of oxygen varies from nothing to 10 per cent. It is more difficult to maintain analgesia with the mouth-piece without wasting much gas, since the depth of anesthesia is now more difficult to control. Oxygen must be used more freely. When the head distends the perineum, the anesthesia is carried to the surgical degree, and the color of the patient is controlled with oxygen. Separate tanks of gas and oxygen are best and cheapest. Their small size admits of easy transportation. Separate tanks permit the variations of percentage of oxygen. With these small tanks the method costs from four to five dollars per hour, varying with the duration and frequency of the pains and the skill of the operator.

Gas is the ideal drug for conducting labors. It is the most volatile of anesthetics, acts most quickly, and its effects pass away most rapidly. It is practically free from danger even when continued for analgesia of many hours. This may be evinced from the work of Woodyatt. ${ }^{2}$ A number of dogs were sensitized to toxins by removal of their glycogen. No animal thus treated survived ten minutes of chloroform narcosis. There were marked changes in the secretions of the dogs anesthetized for the same period with ether. Yet two hours of anesthesia with nitrous oxid caused no demonstrable tissue injuries, and the changes in the secretions were trivial or nil. Theoretically, therefore, we may expect to continue analgesia throughout the entire labor, with trivial resultant injury. Nor does the drug cause, as chloroform, lesions of the fetus, since there is no resultant tissue asphyxia on which are dependent the visceral and hemorrhagic diseases of the new-born (Graham) ${ }^{3}$

This method is not original with me, and was first seen July 12,1913, in a case which I saw with my colleague, Dr. Junius Hoag. The patient was the daugh. ter of Mr. Clark, a maker of instruments for anesthesia, who had sought at his own risk to try in his family the method which his demonstrators were teaching for dentistry. The analgesia was maintained for four hours, with anesthesia to surgical degree, for purpose of delivery. The method was also independently developed by Dr. Heaney about six months later, who tells me he has a considerable series of cases about ready for report. $\mathrm{He}$ is likewise most enthusiastic over the method. ${ }^{4}$

I am of the belief that this will make the use of scopolamin-morphin unnecessary in the treatment of private cases. Its ease of administration and freedom from danger speak volumes for its popularity. Nor is the technic complicated or the good results limited to one man. Unlike the Freiburg method, it is adapted to work in the private home, and is devoid of its many dangers.

104 South Michigan Avenue.

1. Lynch: Jour. Michigan State Med. Soc., 191

2. Woodyatt: Tr. Soc. Exper. Biol. and Med., March, 1914

3. Graham, Evarts: Jour. Exper. Med., April, 1912

4. Personal communication to the author 